\title{
CHOOSING THE BEST PERFORMING GARCH MODEL FOR SRI LANKA STOCK MARKET BY NON-PARAMETRIC SPECIFICATION TEST
}

\author{
Aboobacker Jahufer ${ }^{1 *}$ \\ ${ }^{1}$ South Eastern University of Sri Lanka
}

Abstract:This paper examines the performance of different kind of GARCH models with Gaussian, Student-t and generalized error distribution for Colombo Stock Exchange (CSE), in Sri Lanka. Analyzing the daily closing price index of CSE from January 02, 2007 to March 10, 2013. It was found that the Asymmetric GARCH models give better result than symmetric GARCH model. According to distributional assumption these models under Student-t as well as generalized error provided better fit than normal distributional assumption. The Non-Parametric Specification test suggest that the GARCH, EGARCH, TARCH and APARCH models with Student-t distributional assumption are the most successful model for CSE.

Key words: GARCH Model, Asymmetric GARCH Model, Generalized Error Density, Colombo Stock Exchange, Non Parametric Specification Test.

\section{Introduction}

The various well-known characteristics are common to many financial time series. Volatility clustering is often observed (i.e. large changes tend to be followed by large changes and small changes tend to be followed by small changes; Mandelbrot (1963) for early evidence). Second, financial time series often exhibit leptokurtosis, meaning that the distribution of their returns is fat-tailed see Fama (1965). Moreover, the so-called "leverage effect", first noted in Black (1976), refers to the fact that changes in stock prices tend to be negatively correlated with changes in volatility.

Engle (1982) proposed to model time-varying conditional variance with the Auto Regressive Conditional Heteroskedasticity (ARCH) processes that used past disturbances to model the variance of the series. Early empirical evidence showed that high ARCH order has to be selected in order to catch the dynamic of the conditional variance. The Generalized ARCH (GARCH) model of Bollerslev (1986) was an answer to this issue. It was based on an infinite $\mathrm{ARCH}$ specification and it allows reducing the number of estimated parameters from $\infty$ to only 2 . Both models allow taking the first two characteristics into account but their distribution is symmetric and fat tails therefore,

\footnotetext{
${ }^{*}$ Corresponding author.
} 
458 CHOOSING THE BEST PERFORMING GARCH MODEL FOR SRI LANKA STOCK MARKET BY NON-PARAMETRIC SPECIFICATION TEST

to model the third stylized fact, namely the "leverage effect". To solve this problem, many nonlinear extensions of the GARCH models have been proposed. Among the most widely spread are the Exponential GARCH (EGARCH) of Nelson (1991), The Threshold GARCH (TGARCH) model of Zakoian (1994), Glosten et al. (1993) and the Asymmetric Power ARCH (APARCH) of Ding et al. (1993). Unfortunately, GARCH models often do not fully capture the thick tails property of high frequency financial time series. This has naturally led to the use of non-normal distributions to better model this excess kurtosis. Bollerslev (1986), Baillie and Bollerslev (1989), Kaiser (1996) and Beine, et al. (2000) among others used Student-t distribution while Nelson (1991) and Kaiser (1996) suggested the Generalized Error Distribution (GED). Other propositions include mixture distributions such as the normal-lognormal (Hsieh 1989 or the Bernoulli-normal Vlaar and Palm 1993). Peters (2001) in his working paper showed the forecasting performance of different kinds of asymmetric GARCH model with normal, Student-t and skewed Student-t distributional assumption for two major European stock indices. Forecasting conditional variance with asymmetric GARCH models has been studied in several papers Pagan and Schwert (1990), Brailsford and Faff (1996), Franseset et al. (1998), Loudon et al. (2000). On the other hand, comparing normal density with non-normal ones also has been explored in many occasions Hsieh (1989), Baillie and Bollerslev (1989), Peters (2000) and Lambert and Laurent (2000). Marcucci (2003) compared a number of different GARCH models in terms of their ability to describe and forecast the behavior of volatility of financial time series. He estimated assuming both Gaussian innovations and fat-tailed distributions, such as student- and Generalized Error distribution. Hong and Li (2004) studied the Nonparametric Specification Tests of Discrete Time Spot Interest Rate Models in China. They examined a wide variety of popular spot rate models in China, including the single-factor diffusion models, GARCH models, Markov regimeswitching models and jump-diffusion models and the specification of these models. Hong and Li (2005) conducted a study on Nonparametric Specification Test for ContinuousTime Models with Applications to Spot Interest Rates. Batra (2004) examined the time varying pattern of stock return volatility in Indian stock market. In this study the daily closing index of CSE in Sri Lanka is used. The structure of the assignment is as follows: Section 2 presents the different GARCH models used in this study, densities are described in Section 3 and Section 4 present non-parametric specification test, Section 5 reports the empirical findings while Section 6 present the conclusions.

\section{Models of the Study}

Let us consider a univariate time series ${ }^{y_{t}}$. If $\psi_{t-1}$ is the information set (i.e. all the information available) at time $t-1$, we can define its functional form as:

$$
y_{t}=E\left[y_{t} \mid \psi_{t-1}\right]+\varepsilon_{t}
$$


where $E[. \mid$.$] denotes the conditional expectation operator and \varepsilon_{t}$ is the disturbance term (or unpredictable part), with $E\left[\varepsilon_{t}\right]=0$ and $E\left[\varepsilon_{t} \varepsilon_{s}\right]=0, \forall t \neq s$.

The ${ }^{\varepsilon_{t}}$ term in Eq. (1) is the innovation of the process. The conditional expectation is the expectation conditional to all past information available at time $t-1$. The Autoregressive Conditional Heteroscedastic (ARCH) process of Engle (1982) is any $\left\{\varepsilon_{t}\right\}$ of the form

$$
\varepsilon_{t}=z_{t} \sigma_{t}
$$

where $z_{t}$ is independently and identically distributed (i.i.d.) process, $E\left[z_{t}\right]=0$, $\operatorname{Var}\left[z_{t}\right]=1$ and where ${ }^{\sigma_{t}}$ is a time-varying positive and measurable function of the information set at time $t-1$. By definition, ${ }^{\varepsilon_{t}}$ is serially uncorrelated with mean zero but its conditional variance equals to $\sigma_{t}^{2}$ and therefore, may change over time, contrary to what is assumed in OLS estimations. Specifically the ARCH (q) model is given by

$$
\sigma_{t}^{2}=\alpha_{0}+\sum_{i=1}^{q} \alpha_{i} \varepsilon_{t-i}^{2} .
$$

The models considered in this paper are all ARCH-type. They differ on the functional form of $\sigma_{t}^{2}$ but the basic logic is the same.

\subsection{GARCH Model}

The GARCH model of Bollerslev (1986) can be expressed as

$$
\sigma_{t}^{2}=\alpha_{0}+\sum_{i=1}^{q} \alpha_{i} \varepsilon_{t-i}^{2}+\sum_{j=i}^{p} \beta_{j} \sigma_{t-j}^{2} .
$$

Using the lag or backshift operator $L$, the $\operatorname{GARCH}(\mathrm{p}, \mathrm{q})$ model is

$$
\sigma_{t}^{2}=\alpha_{0}+\alpha(L) \varepsilon_{t}^{2}+\beta(L) \sigma_{t}^{2}
$$

with $\alpha(L)=\alpha_{1} L+\alpha_{2} L^{2}+\ldots .+\alpha_{q} L^{q}$ and $\beta(L)=\beta_{1} L+\beta_{2} L^{2}+\ldots .+\beta_{p} L^{p}$.

Based on Eq. (5), it is straightforward to show that the GARCH model is based on an infinite ARCH specification. If all the roots of the polynomial $1-\beta(L)=0$ of Eq. (5) lie outside the unit circle, we have $\sigma_{t}^{2}=\alpha_{0}[1-\beta(L)]^{-1}+\alpha(L)[1-\beta(L)]^{-1} \varepsilon_{t}^{2}$ or equivalently 


$$
\sigma_{t}^{2}=\frac{\alpha_{0}}{1-\beta_{1}-\ldots \ldots . \beta_{p}}+\sum_{i=1}^{\infty} \lambda_{i} \varepsilon_{t-i}^{2}
$$

, which may be seen as an $\mathrm{ARCH}(\infty)$ process since the conditional variance linearly depends on all previous squared residuals.

\subsection{EGARCH Model}

The EGARCH or Exponential GARCH model was proposed by Nelson (1991). The specification for conditional variance is:

$$
\log \left(\sigma_{t}^{2}\right)=\alpha_{0}+\sum_{j=1}^{q} \beta_{j} \log \left(\sigma_{t-j}^{2}\right)+\sum_{i=1}^{p} \alpha_{i}\left|\frac{\varepsilon_{t-i}}{\sigma_{t-i}}\right|+\sum_{k=1}^{r} \gamma_{k} \frac{\varepsilon_{t-k}}{\sigma_{t-k}}
$$

The left hand side of Eq. (6) is the log of the conditional variance. This implies that the leverage effect is exponential rather than quadratic and that forecasts of the conditional variance are guaranteed to be non-negative. The presence of the leverage effects can be tested by the hypothesis $\gamma_{i}<0$. The impact is asymmetric if $\gamma_{i} \neq 0$.

\subsection{TGARCH Model}

TARCH or Threshold ARCH and Threshold GARCH were introduced independently by Zakoian (1994) and Glosten et al. (1993). The generalized specification for the conditional variance is given by:

$$
\sigma_{t}^{2}=\alpha_{0}+\sum_{j=1}^{q} \beta_{j} \sigma_{t-j}^{2}+\sum_{i=1}^{p} \alpha_{i} \varepsilon_{t-i}^{2}+\sum_{k=1}^{r} \gamma_{k} \varepsilon_{t-k}^{2} \Gamma_{t-k}
$$

where $\Gamma_{t}=1$, if $\varepsilon_{t}<0$ or $\Gamma_{t}=0$, otherwise.

In this model $\varepsilon_{t-i}>0$ and $\varepsilon_{t-i}<0$ have differential effects on the conditional variance; $\varepsilon_{t-i}>0$ has an impact of $\alpha_{i}$, while $\varepsilon_{t-i}^{<0}$ has an impact of $\alpha_{i}+\gamma_{i}$. If $\gamma_{i}>0, \varepsilon_{t-i}<0$

increases volatility and we say that there is a leverage effect for the $i-t h$ order. If $\gamma_{i} \neq 0$ the news impact is asymmetric.

\subsection{APARCH Model}

Taylor (1986) and Schwert (1990) introduced the standard deviation GARCH model where the standard deviation is modeled rather than the variance. Ding, et al. (1993) introduced the Asymmetric Power ARCH model. The APARCH (p, q) model can be expressed as: 


$$
\sigma_{t}^{\delta}=\alpha_{0}+\sum_{j=1}^{p} \beta_{j} \sigma_{t-j}^{\delta}+\sum_{i=1}^{q} \alpha_{i}\left(\left|\varepsilon_{t-i}\right|-\gamma_{i} \varepsilon_{t-i}\right)^{\delta}
$$

where $\alpha_{0}>0, \delta \geq 0, \beta_{j} \geq 0(j=1,2, \ldots \ldots ., p), \alpha_{i} \geq 0$ and $-1<\gamma_{i}<1,(i=1,2, \ldots, q)$.

This model is quite interesting since it couples the flexibility of a varying exponent with the asymmetry coefficient (to take the "leverage effect" into account). Moreover, the APARCH model includes seven other ARCH extensions as a special case:

i) ARCH when $\delta=2, \gamma_{i}=0(i=1,2, \ldots . p)$ and $\beta_{j}=0(j=1,2, \ldots . p)$.

ii) GARCH when $\delta=2, \gamma_{i}=0(i=1,2, \ldots . p)$.

iii) Taylor (1986) / Schwert (1990) GARCH when $\delta=1, \gamma_{i}=0(i=1,2, \ldots . p)$.

iv) GJR when $\delta=2$, v) TARCH when $\delta=1$.

vi) NARCH when $\gamma_{i}=0(i=1,2, \ldots . p)$ and $\beta_{j}=0(j=1,2, \ldots . p)$.

vii) The log-ARCH of Geweke (1986) and Pentula (1986), whenever $\delta>0$.

\section{Distributional Assumptions}

It may be expected that excess kurtosis and skewness displayed by the residuals of conditional heteroscedasticity model will be reduced if a more appropriate distribution is used. In this study, three distributions such as Gaussian, Student-t and Generalized Error are considered. Given a distributional assumption, ARCH models are typically estimated by the method of maximum likelihood.

\subsection{Gaussian}

The Normal distribution is by far the most widely used distribution when estimating and forecasting GARCH models. If we express the mean equation as in Eq. (1) and $\varepsilon_{t}=z_{t} \sigma_{t}$, the log-likelihood function of the standard normal distribution is given by

$$
L_{T}=-\frac{1}{2} \sum_{t=1}^{T}\left[\ln (2 \pi)+\ln \left(\sigma_{t}^{2}\right)+z_{t}^{2}\right]
$$

where $T$ is the number of observations.

\subsection{Student t-Distribution}

For the Student-t distribution, the log-likelihood contributions are of the form: 


$$
\begin{aligned}
L_{T}=\ln \left[\sqrt{\left(\frac{v+1}{2}\right)}\right]-\ln \left[\sqrt{\left(\frac{v}{2}\right)}\right]-0.5 \ln [\pi(v-2)] \\
-0.5 \sum_{t=1}^{T}\left[\ln \left(\sigma_{t}^{2}\right)+(1+v) \ln \left(1+\frac{z_{t}^{2}}{v-2}\right)\right]
\end{aligned}
$$

where $v$ is the degree of freedom $2<v \leq \infty$ and $\sqrt{(.)}$ is the gamma function. When $v \rightarrow \infty$, we have the normal distribution. So that the lower $v$ the fatter the tails.

\subsection{Generalized Error}

For the GED, we have:

$$
L_{T}=-\frac{1}{2} \ln \left[\frac{\sqrt{(1 / r)^{3}}}{\sqrt{(3 / r)(r / 2)^{2}}}\right]-0.5 \ln \left(\sigma_{t}^{2}\right)-\left[\frac{\sqrt{(3 / r)} z_{t}^{2}}{\left.\sigma_{t}^{2}\right)(1 / r)}\right]^{r / 2}
$$

where the tail parameter $r>0$. The GED is a normal distribution if $r=2$, and fat-tailed if $r<2$.

\section{Non Parametric Specification Test}

Recently Hong and Li (2005) proposed two new nonparametric transition density-based specification tests for continuous-time series models. These tests share the appealing features of both Ait-Sahalia (1996) and Gallant and Tauchen (1996) nonparametric approaches and have many additional nice properties. First, unlike Ait-Sahalia (1996) marginal density-based test, the tests are based on the transition density which captures the full dynamics of a continuous-time process. Second, to achieve robustness to persistent dependence, the data is transformed via a dynamic probability integral transform using the model transition density which is well known in statistics (e.g., Rosenblatt 1952) and is more recently used to evaluate out-of-sample density forecasts in discrete-time analysis (e.g. Diebold, et al. 1998, Hong et al. 2004). The transformed sequence is i.i.d. $\mathrm{U}[0,1]$ under correct model specification irrespective of the dependence structure of the original data. Third, to eliminate the well-known "boundary bias" of kernel estimators as documented in Chapman and Pearson (2000), a boundary-modified kernel is introduced. Fourth, to reduce the impact of parameter estimation uncertainty, a test based on the Hollinger metric is proposed. Fifth, the regularity conditions for asymptotic analysis are based on the model transition density rather than the stochastic differential equation of the underlying process. As a consequence, the tests are applicable to a vast variety of continuous-time and discrete-time dynamic models such as GARCH/stochastic volatility models, regime-switching models, jump-diffusion models and multi-factor diffusion models. The nonparametric 
specification test proposed recently by Hong and $\mathrm{Li}$ (2005) to evaluate different spot rate models. Assuming the underlying process $\{\mathrm{Xt}\}$ follows the following data generating process:

$$
d X_{t}=\mu_{0}\left(X_{t}, t\right) d t+\sigma_{0}\left(X_{t}, t\right) d W_{t}
$$

where $\mu_{0}\left(X_{t}, t\right)$ and $\sigma_{0}\left(X_{t}, t\right)$ are the drift and diffusion functions respectively and $W_{t}$ is a standard Brownian motion. Let $p_{0}(x, t / y, s)$ be the true transition density of the diffusion process $\left\{X_{t}\right\}$ that is the conditional density of $\left(X_{t}=x, X_{s}=y\right), s<t$. For a given

pair of drift and diffusion models $\mu\left(X_{t}, t, \theta\right)$ and $\sigma\left(X_{t}, t, \theta\right)$ a certain family of transition densities $\{p(x, t / y, s, \theta)\}$ is characterized. If a model is correctly specified, there exists some $\theta_{0} \in \Theta_{\text {satisfying }}\left\{p\left(x, t / y, s, \theta_{0}\right)=p_{0}(x, t / y, s)\right\}$ almost everywhere for some $\theta_{0} \in \Theta$. To test such a hypothesis, Hong and Li (2005) first transform the discretized data $\left\{X_{\tau \Delta}\right\}_{\tau=1}^{n}$ via a probability integral transform and define this discrete transformed sequence by

$$
Z_{\tau}(\theta) \equiv \int_{-\infty}^{X_{\tau \Delta}} p\left[x, \tau \Delta / X_{(\tau-1) \Delta},(\tau-1) \Delta, \theta\right] d x, \tau=1,2, \ldots \ldots, n .
$$

If the model is correctly specified, the exists some $\theta_{0} \in \Theta$ such that $p\left[x, \tau \Delta / X_{(\tau-1) \Delta},(\tau-1) \Delta, \theta_{0}\right]=p_{0}\left[x, \tau \Delta / X_{(\tau-1) \Delta},(\tau-1) \Delta\right]$ almost surely for all $\Delta>0$.

Consequently, the transformed series $\left\{Z_{\tau} \equiv Z_{\tau}\left(\theta_{0}\right)\right\}_{\tau=1}^{n}$ is i.i.d. $U[0,1]$ under correct specification. It can be called that $\left\{Z_{\tau}(\theta)\right\}_{\tau=1}^{n}$ "generalized residuals" of the model $\{p(x, t / y, s, \theta)\}$. Here, i.i.d $U[0,1]$ property captures two important aspects of model specification, i.i.d characterize the correct specification of model dynamics and $U[0,1]$ characterize correct specification of the model marginal distribution. The test that whether $\left\{Z_{\tau}(\theta)\right\}_{\tau=1}^{n}$ follows i.i.d $U[0,1]$ is not a trivial task because it is a joint hypothesis. The wellknown Kolmogorov-Smirnov test checks $U[0,1]$ under the i.i.d assumption rather than test i.i.d and $U[0,1]$ jointly. It would miss the alternatives whose marginal distribution is uniform but not i.i.d. To make such joint hypothesis tests, Hong and Li (2005) develop two nonparametric tests of i.i.d. $U[0,1]$ by comparing a kernel estimator $\hat{g}_{j}\left(z_{1}, z_{2}\right)$ for the joint density $\hat{g}_{j}\left(z_{1}, z_{2}\right)$ of $\left\{Z_{\tau}, Z_{\tau-j}\right\}$ with unity, the product of two $U[0,1]$ densities. The kernel joint density estimator is for any integer $j>0$. 
NON-PARAMETRIC SPECIFICATION TEST

$$
\hat{g}_{j}\left(z_{1}, z_{2}\right)=(n-j)^{-1} \sum_{\tau=j+1}^{n} K_{h}\left(z_{1}, \hat{Z}_{\tau}\right) K_{h}\left(z_{1}, \hat{Z}_{\tau-j}\right)
$$

where, $K_{h}(x, y)=\left\{\begin{array}{l}h^{-1} k\left(\frac{x-y}{h}\right) / \int_{-(x / h)}^{1} k(u) d u, x \in[0, h) \\ h^{-1} k\left(\frac{x-y}{h}\right), x \in[h, 1-h] \\ h^{-1} k\left(\frac{x-y}{h}\right) / \int_{-1}^{(1-x) / h} k(u) d u, x \in[1-h, 1] .\end{array}\right.$

And the kernel $\mathrm{k}($.$) is a bounded symmetric probability density with support [-1,1]$ so that $\int_{-1}^{1} k(u) d u=1, \int_{-1}^{1} u k(u) d u=0$ and $\int_{-1}^{1} u^{2} k(u) d u<\infty$, one choice is the quartic kernel:

$$
k(u)=\frac{15}{16}\left(1-u^{2}\right) \sum_{i=1}^{n} X_{1}^{2} 1(|u| \leq 1)
$$

where $1(|u| \leq 1)$ is the indicator function, taking value 1 if $|u| \leq 1$ and zero, otherwise. $\hat{Z}_{\tau}=Z_{\tau}(\hat{\theta})$ and $\hat{\theta}_{\text {is a }} \sqrt{n}$ - consistent estimator for $\theta_{0} . h=\hat{S}_{z} n^{-1 / 6}$, where $\hat{S}_{z}$ is the sample standard deviation of $\left\{Z_{\tau}\right\}_{\tau=1}^{n}$. The first tests is based on a quadratic form between $\hat{g}_{j}\left(z_{1}, z_{2}\right)$ and 1 , the product of two $U[0,1]$ densities,

$$
\hat{M}_{1}(j) \equiv \int_{0}^{1} \int_{0}^{1}\left[\hat{g}_{j}\left(z_{1}, z_{2}\right)-1\right]^{2} d z_{1} d z_{2}
$$

and the first test statistic is a properly centered and scaled version of $\hat{M}_{1}(j)$ :

$$
\hat{Q}(j) \equiv\left[(n-1) h \hat{M}_{1}(j)-A_{h}^{0}\right] / V_{0}^{1 / 2},
$$

where the non-stochastic centering and scale factors

$$
\begin{gathered}
A_{h}^{0} \equiv\left(h^{-1}-2\right) \int_{-1}^{1} k^{2}(u) d u+\int_{0}^{1} \int_{-1}^{b} k_{b}^{2}(u) d u d b, \\
V_{0} \equiv 2\left[\int_{-1}^{1}\left[\int_{-1}^{1} k(u+v) k(v)\right]^{2} d u\right]^{2}
\end{gathered}
$$

$$
\text { and } k_{b}(.) \equiv k(.) / \int_{-1}^{b} k(v) d v
$$


Under correct model specification, (Hong and Li 2005, Theorem 1) has shown that $\hat{Q}(j) \rightarrow N(0,1)$ in distribution and under model misspecification, $\hat{Q}(j) \rightarrow \infty$ in probability. Whenever $\left\{Z_{\tau}, Z_{\tau-j}\right\}$ are not independent or $U[0,1]$. (Hong and Li 2005, Theorem 3).

The quadratic form test $\hat{Q}(j)$ though convenient and quite accurate when the true parameter $\theta_{0}$ was known might be adversely affected by any imprecise estimate for $\hat{\theta}$ in finite samples. To alleviate this problem they proposed a second test based on the square Hellinger metric,

$$
\hat{M}_{1}(j) \equiv \int_{0}^{1} \int_{0}^{1}\left[\sqrt{\hat{g}_{j}\left(z_{1}, z_{2}\right)}-1\right]^{2} d z_{1} d z_{2}
$$
is

which is a quadratic form between $\sqrt{\hat{g}_{j}\left(z_{1}, z_{2}\right)}$ and $\sqrt{1.1}=1$. The associated test statistic

$$
\hat{H}(j) \equiv\left[4(n-j) h \hat{M}_{2}(j)-A_{h}^{0}\right] / V_{0}^{1 / 2},
$$

where $A_{h}^{0}$ and $V_{0}$ are as in Eq. (18) and Eq. (19). Under correct model specification this test has the same asymptotic distribution as $\hat{Q}(j)$ and is asymptotically equivalent to $\hat{Q}(j)$ in the sense that $\hat{Q}(j)-\hat{H}(j) \rightarrow 0$ in probability. Under model misspecification we also have $\hat{H}(j) \rightarrow \infty$ as $\mathrm{n} \rightarrow \infty$ whenever $\left\{Z_{\tau}, Z_{\tau-j}\right\}$ are not independent or $U[0,1]$.

It can be summarized the omnibus evaluation procedures following Hong and Li (2005): (i) estimate the parameters of discrete spot rate models using maximum likelihood estimation (MLE) method to yield a $\sqrt{n}$-consistent estimator $\hat{\theta}$; (ii) compute the model generalized residual $\left\{\hat{Z}_{\tau}=Z_{\tau}(\hat{\theta})\right\}_{\tau=1}^{n}$, where $Z_{\tau}(\theta)$ is given in Eq. (13); (iii) compute the boundary- modified kernel joint density estimator $\hat{g}_{j}\left(z_{1}, z_{2}\right)$ in Eq. (14) for a pre-specified lag j , using a kernel in Eq. (15) and the bandwidth $h=\hat{S}_{z} n^{-1 / 6}$, where $\hat{S}_{z}$ is the sample standard deviation of the model generalized residual $\left\{\hat{Z}_{\tau}\right\}_{\tau=1}^{n}$; (iv) compute the test statistics $\hat{Q}(j)$ in Eq. (17) and $\hat{H}(j)$ in Eq. (21); (v) compare the value of $\hat{Q}(j)$ or $\hat{H}(j)$ with the upper-tailed $N(0,1)$ critical value $C_{\alpha}$ at level $\alpha$ (e.g., $C_{0.05}=1.645$ ). The upper-tailed rather than two sided $N(0,1)$ critical values is suitable since negative $\hat{Q}(j)$ and $\hat{H}(j)$ occurs only under correct model specification when $n$ is sufficiently large. Both of $\hat{Q}(j)$ and $\hat{H}(j)$ diverge to $+\infty$ when $\left\{Z_{\tau}, Z_{\tau-j}\right\}$ are not independent or $U[0,1]$ under model specification granting the tests asymptotic unit power. 


\section{Empirical Study}

The data analyzed here is the daily closing price index for CSE in Sri Lanka from January 02, 2007 to March 10, 2013. The parameter estimation process choice here is MLE. The indices prices are transformed into their returns to obtain stationary series. The transformation is: $r_{t}=100 *\left[\ln \left(y_{t}\right)-\ln \left(y_{t-1}\right)\right]$, where ${ }^{y}$ is the return index at time ${ }^{t}$. Refer table 1 .

Some of the descriptive statistics for daily returns are displayed in Table 1. Mean returns of the CSE is 0.067 . Volatility (measured as a standard deviation) is 1.6012. The return of CSE market is leptokurtic in the sense that kurtosis exceeds positive three and the return series also display significant skewness. According to Jarque and Bera (1987) statistics normality is rejected for the return series. The results from ARCH test can not reject the ARCH effect which indicate that the series have ARCH effect. Overall these results clarify support the rejection of the hypothesis that CSE time series of daily returns are time series with independent daily values. Moreover, the statistics justify use of the GARCH specification in modeling the volatility of Sri Lanka stock market.

Table 1: Descriptive Statistics of CSE daily returns.

\begin{tabular}{|l|l|l|l|l|l|l|l|l|}
\hline $\begin{array}{l}\text { Sample } \\
\text { Size }\end{array}$ & Mean & Min. & Max. & $\begin{array}{l}\text { Standard } \\
\text { Deviation }\end{array}$ & Skewness & Kurtosis & $\begin{array}{l}\text { Jarque-Bera (JB) } \\
\text { Test (p-value) }\end{array}$ & ARCH Test \\
\hline 1550 & 0.067 & -20.568 & 29.214 & 1.6012 & 4.174 & 144.16 & $1298067(0.00)$ & $0.0012(0.97)$ \\
\hline
\end{tabular}

\section{Parameter Estimation}

In comparison of the performance of GARCH models the simple mean equation is used:

$y_{t}=\mu+\varepsilon_{t}$ for all models.

Table 2: MLE of the parameters and their corresponding t-statistic

\begin{tabular}{|c|c|cccccccc|}
\hline \multicolumn{2}{|c}{} & & $\alpha_{0}$ & $\alpha_{i}$ & $\beta_{j}$ & $\gamma_{k}$ (or $\left.\gamma_{i}\right)$ & $v$ & $r$ & $\delta$ \\
\hline \multirow{4}{*}{1} & $\mathrm{~N}$ & 0.1800 & 1.092 & 0.3792 & 0.2813 & & & \\
& & $\mathrm{~S}$ & $(4.568)$ & $(13.632)$ & $(6.571)$ & $(5.526)$ & & & \\
& & 0.0019 & 0.1460 & 0.7099 & 0.3388 & & 3.8813 & \\
& & $(0.1301)$ & $(7.448)$ & $(7.528)$ & $(8.754)$ & & $(17.944)$ & \\
& $\mathrm{G}$ & 0.0122 & 0.2390 & 0.8200 & 0.2776 & & & 0.8150 \\
& & $(1.921)$ & $(8.722)$ & $(5.912)$ & $(61.278)$ & & & $(61.278)$ \\
\hline & $\mathrm{N}$ & 0.1529 & -0.0505 & -0.3430 & 0.6991 & 0.5058 & & \\
& & $(4.381)$ & $(-4.633)$ & $(-6.558)$ & $(10.826)$ & $(12.613)$ & & \\
2 & $\mathrm{~S}$ & 0.0052 & -0.3620 & 0.1203 & 0.4360 & 0.7338 & 3.350 & \\
& & $(0.3201)$ & $(-8.171)$ & $(3.124)$ & $(8.059)$ & $(20.441)$ & $(16.365)$ & \\
& $\mathrm{G}$ & 0.0124 & -0.3846 & -0.1510 & 0.5078 & 0.6382 & & 0.8043 \\
& & $(1.820)$ & $(7.284)$ & $(-3.172)$ & $(7.359)$ & $(11.840)$ & & $(53.979)$ & \\
\hline & $\mathrm{N}$ & 0.2016 & 0.9237 & 0.8529 & 0.3085 & 0.2500 & & \\
& & $(4.685)$ & $(15.068)$ & $(4.5751)$ & $(6.780)$ & $(6.527)$ & & \\
\end{tabular}


Aboobacker Jahufer

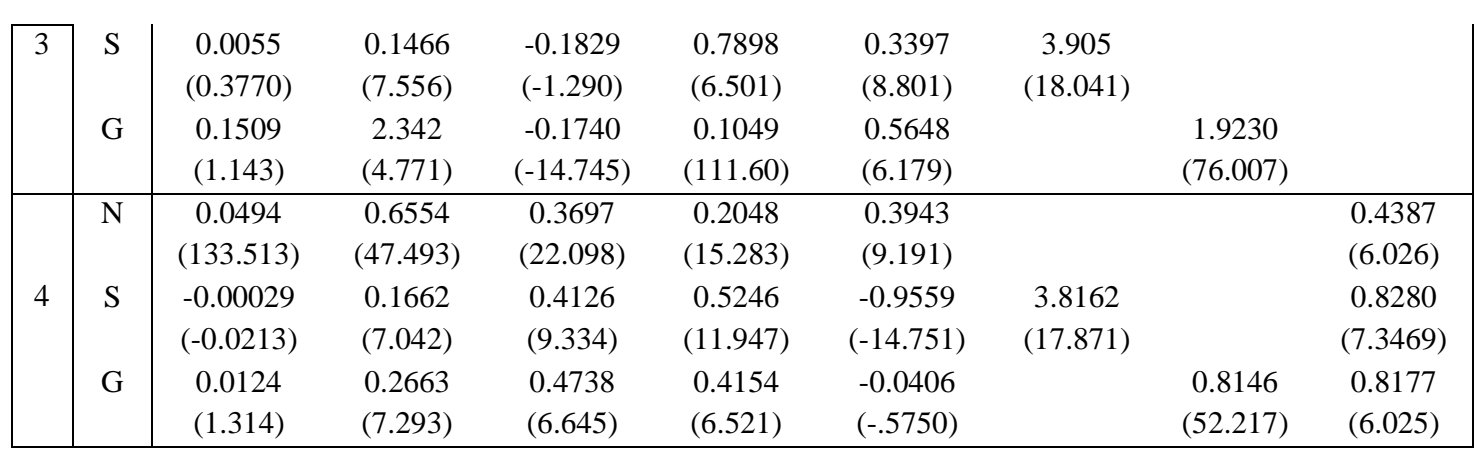

[Note: 1, 2, 3 and 4 represent GARCH, EGARCH, TGARCH and APARCH model respectively and N, S, G indicate Gaussian, Student-t and Generalized error distribution respectively]

Table 2 presents the maximum likelihood estimate (MLE) of the parameters and their corresponding t-statistic. The use of GARCH set of models seems to be justified. All coefficients are significant at $5 \%$ level of significance except the coefficient of ${ }^{\mu}$ in case of Student-t and generalized error densities. Table 3 reports some useful statistics such as Box-Pierce statistics for both residuals and the squared residuals, Akaike Information Criteria (AIC) and Log Likelihood value.

Table 3: Estimation Statistics-Model comparison

\begin{tabular}{|c|c|c|c|c|c|}
\hline & & $Q(20)$ & $Q^{2}(20)$ & AIC & Log-likelihood \\
\hline \multirow{4}{*}{ Gaussian } & GARCH & 10.669 & 2.2003 & 3.4867 & -2712.20 \\
\hline & EGARCH & 13.395 & 2.2461 & 3.4504 & -2682.90 \\
\hline & TGARCH & 9.3922 & 2.2584 & 3.4749 & -2702.01 \\
\hline & APARCH & 11.281 & 2.0266 & 3.4440 & -2677.56 \\
\hline \multirow{4}{*}{ Student-t } & GARCH & 6.568 & 1.9802 & 2.3598 & -1855.10 \\
\hline & EGARCH & 9.7426 & 2.2565 & 2.3123 & -1795.31 \\
\hline & TGARCH & 6.4787 & 2.9364 & 2.2990 & -1754.41 \\
\hline & APARCH & 5.9830 & 1.8125 & 2.2453 & -1742.14 \\
\hline \multirow{4}{*}{ GED } & GARCH & 7.0015 & 2.2057 & 2.4675 & -1907.11 \\
\hline & EGARCH & 11.6570 & 2.2602 & 2.4069 & -1868.97 \\
\hline & TGARCH & 19.1910 & 2.3436 & 2.4635 & -1898.72 \\
\hline & APARCH & 6.9929 & 0.2241 & 2.3676 & -1837.38 \\
\hline
\end{tabular}

The Akaike Information Criteria (AIC) and the log-likelihood values suggest the fact that TGARCH, EGARCH or APARCH models fit the data well than the traditional GARCH. On the basis of AIC and log-likelihood value the APARCH model outperforms than TGARCH and EGARCH model. EGARCH model gives better result than TGARCH model in case of Gaussian and Generalized error densities. Regarding the densities, the Student-t and Generalized error 
468 CHOOSING THE BEST PERFORMING GARCH MODEL FOR SRI LANKA STOCK MARKET BY NON-PARAMETRIC SPECIFICATION TEST

distributions clearly outperform the Gaussian. In the case of Student-t distribution the AIC value for the model GARCH, EGARCH, TGARCH and APARCH are less than the densities of Gaussian density. The log likelihood value is strictly increasing in case of Student-t densities comparing with the generalized error and Gaussian densities. All the models seem to do a good job in describing the dynamic of the first two moments of the series as shown by the Box-Pierce statistics for the residuals and the squared residuals which are all non-significant at $5 \%$ level.

Table 4: Models with Gaussian distributional assumption

\begin{tabular}{|lllll|}
\hline J & GARCH & EGARCH & TGARCH & APARCH \\
\hline 1 & 166.45 & 158.96 & 165.21 & 160.78 \\
5 & 150.12 & 144.23 & 104.95 & 148.47 \\
10 & 138.56 & 130.45 & 98.01 & 130.14 \\
15 & 125.47 & 120.45 & 80.24 & 118.10 \\
\hline
\end{tabular}

Table 5: Models with student t- distributional assumption

\begin{tabular}{|lllll|}
\hline J & GARCH & EGARCH & TGARCH & APARCH \\
\hline 1 & 10.42 & 9.28 & 8.48 & 10.11 \\
5 & 0.95 & 0.88 & 0.86 & 0.84 \\
10 & 0.51 & 0.21 & 0.40 & 0.46 \\
15 & -1.52 & -1.49 & -1.27 & -1.34 \\
\hline
\end{tabular}

Table 6: Models with generalized error distributional assumption

\begin{tabular}{|ccccc|}
\hline J & GARCH & EGARCH & TGARCH & APARCH \\
\hline 1 & 96.47 & 88.36 & 79.63 & 89.52 \\
5 & 78.45 & 71.25 & 58.66 & 70.56 \\
10 & 66.32 & 43.14 & 52.99 & 62.52 \\
15 & 40.38 & 30.58 & 32.66 & 35.42 \\
\hline
\end{tabular}

To identify the best performing model in this section, to do the specification tests follow the test procedures of Hong and $\mathrm{Li}$ (2005) and compute the relevant $\widehat{Q}_{(\mathrm{j}) \text { stats and picking up } j}$ from 1 to 15 . In this paper, it has been taken only $1,5,10$ and 15 as the value of $j$ to calculate the value of $\hat{Q}(j)$ from the each class of volatility rate models, (it's mentionable that the results of $\hat{H}(\mathrm{j})$ tests are quite similar). 
Table 4 reports the $\widehat{Q}(j)$ test statistics as function of lag order ${ }^{j}=1,5,10,15$ for these models with Gaussian distributional assumptions. As shown in the table 4 the $\widehat{Q}(j)$ statistics for the GARCH, EGARCH, TGARCH and APARCH model under Gaussian distribution ranges from 80.24 to 166.45 . Compared with the upper tailed $N(0,1)$ critical value (e.g. 2.33 at the $1 \%$ level and 1.66 at the $5 \%$ level), the large $\widehat{Q}(j)$ statistics are overwhelmingly significant, suggesting that all four models are severely mis-specified at any reasonable significance level. Under Student-t distributional assumption the $\widehat{Q}(j)$ statistics for the following four models are given in Table 5 ranges from -1.52 to 10.42 which can pass the original premise on $5 \%$ as well as $1 \%$ level. It means that GARCH, EGARCH, TGARCH and APARCH models under the student- $t$ distributional assumption are the most successful models. Table 6 reports these models with Generalized Error distribution. The $\widehat{Q}_{(\mathrm{j}) \text { statistics for these models are range from } 30.58 \text { to }}$ 96.47. Adding Generalized Error distribution into these models reduces the $\widehat{Q}_{(\mathrm{j}) \text { statistics but }}$ these $\hat{Q}_{(\mathrm{j}) \text { statistics are higher than student- }}{ }^{t}$ distributional assumption. However, compared

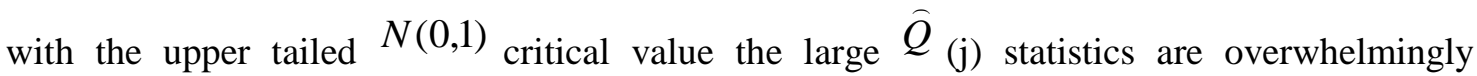
significant, suggesting that all four models are not significance at any reasonable significance level.

\section{Conclusions}

This research paper examined a wide variety of popular GARCH models for CSE index return, including GARCH, EGARCH, TGARCH and APARCH model with Gaussian, Student-t and generalized error distribution. The comparison focused on two different aspects: the difference between symmetric and asymmetric GARCH (i.e., GARCH versus EGARCH, TGARCH and APARCH) and the difference among Gaussian, Student-t and Generalized error densities. The results show that noticeable improvements were made by using an asymmetric GARCH model in the conditional variance. According to distributional assumption these models with Student-t distribution give better results. The Non Parametric Specification test also suggests that the following model under student- $t$ distribution can pass the original premise on $5 \%$ as well as $1 \%$ level. It means the GARCH, EGARCH, TGARCH and APARCH models under Studentt distributional assumption are significant models for CSE. Finally the study suggested that these four models were very suitable for the stock market of Sri Lanka. As a result, this study would be of great benefit to investors and policy makers at home and abroad.

\section{Acknowledgements}


470 CHOOSING THE BEST PERFORMING GARCH MODEL FOR SRI LANKA STOCK MARKET BY

NON-PARAMETRIC SPECIFICATION TEST

The Author would like to thank an anonymous referees for their valuable comments and suggestions. This research work was supported by a research grant No: SEU/EX/RG/128 by South Eastern University of Sri Lanka.

\section{References}

[1] Ait-Sahalia, Y. (1996). Testing Continuous-Time Models of the Spot Interest Rate. Review of Financial Studies 9, 385-426.

[2] Baillie, R. and Bollerslev, T. (1989). The Message in Daily Exchange Rates: A ConditionalVariance Tale. Journal of Business and Economic Statistics 7, 297-305.

[3] Batra, A. (2004). Stock Return Volatility Patterns in India. Working Paper no.124, Indian Council for Research on International Economic Relations, New Delhi, India.

[4] Beine, M. Laurent, S. and Lecourt, C. (2000). Accounting for Conditional Leptokurtosis and Closing Days Effects in FIGARCH Models of Daily Exchange Rates, Applied Financial Economics.

[5] Black, F. (1976). Studies of Stock Market Volatility Changes. Journal of Business and Economic Statistics 16, 177-181.

[6] Bollerslev, T. (1986). Generalized autoregressive conditional heteroskedasticity. Journal of Econometrics 31, 307-327.

[7] Brailsford, T. and Faff, R. (1996). An evaluation of volatility forecasting techniques. Journal of Banking and Finance 20, 419-438.

[8] Chapman, D. and Pearson, N. (2000), Recent Advances in Estimating Models of the TermStructure. Financial Analysts Journal 57, 77-95.

[9] Diebold, F. X. Gunther, T. A. and Tay, A. S. (1998). Evaluating Density Forecasts With Applications to Financial Risk Management. International Economic Review 39, 863-883.

[10] Ding, Z., Granger, C.W.J. and Engle, R.F. (1993). A Long Memory Property of Stock Market Returns and a New Model. Journal of Empirical Finance 1, 83-106.

[11] Engle, R. (1982). Autoregressive conditional heteroscedasticity with estimates of the variance of United Kingdom inflation. Econometrica 50, 987-1007.

[12] Fama, E. (1965). The Behaviour of Stock Market Prices. Journal of Business 38, 34-105.

[13] Franses, P. Neele, J. and Van Dijk, D. (1998). Forecasting volatility with switching persistence GARCH models. Working Paper. Erasmus University, Rotterdam.

[14] Gallant, A.R. and Tauchen, G. (1996). Which Moments to Match?, Econometric Theory 12, 657-681.

[15] Geweke, J. (1986). Modeling the Persistece of Conditional Variances: A Comment. Econometric Review 5, 57-61.

[16] Glosten, L., Jagannathan, R. and Runkle, D. (1993). On the relation between expected return on stocks. Journal of Finance 48, 1779-1801. 
[17] Hong, Y., Li, H. and Zhao, F. (2004). Out-of-Sample Performance of Discrete-Time Spot Interest Rate Models, Journal of Business \& Economics Statistics, Forthcoming

[18] Hong, Y. and Li, H. (2004). Nonparametric Specification Tests of Discrete Time Spot Interest Rate Models in China, An unpublished manuscript.

[19] Hong, Y. and Li, H. (2005). Nonparametric Specification Testing for Continuous-Time Models with Applications to Interest Rate Term Structures. The Review of Financial Studies 18(1): 3784.

[20] Hsieh, D. (1989). Modeling Heteroskedasticity in Daily Foreign Exchange Rates. Journal of Business and Economic Statistics 7, 307-317.

[21] Jarque, C. and Bera, A. (1987). A Test for Normality of Observations and Regression Residuals. International Statistical Review 55, 163-172.

[22] Kaiser, T. (1996). One-Factor-GARCH Models for German Stocks - Estimation and Forecasting. Working Paper, Universiteit Tubingen.

[23] Lambert, P. and Laurent, S. (2000). Modelling Skewness Dynamics in Series of Financial Data. Discussion Paper, Institut de Statistique, Louvain-la-Neuve.

[24] Loudon, G., Watt, W. and Yadav, P. (2000). An empirical analysis of alternative parametric ARCH models. Journal of Applied Econometrics 15, 117-136.

[25] Mandelbrot, B. (1963). The Variation of Certain Speculative Prices. Journal of Business 36, 394-419.

[26] Marcucci, J. (2003). Forecasting Stock Market Volatility with Regime-Switching GARCH Models. Department of Economics, University of California, San Diego, USA.

[27] Nelson, D. (1991). Conditional heteroskedasticity in asset returns: a new approach. Econometrica 59, 349-370.

[28] Pagan, A. and Schwert, G. (1990). Alternative Models for Conditional Stock Volatility. Journal of Econometrics 45, 267-290.

[29] Pentula, S. (1986). Modeling the Persistece of Conditional Variances: A Comment. Econometric Review 5, 71-74.

[30] Peters, J.P. (2001). Estimating and forecasting volatility of stock indices using asymmetric GARCH models and (skewed) Student-t densities. Working paper, E'cole $d^{\prime}$ Administration des affaires, University of Li Lie'ge, Belgium, March 20.

[31] Peters, J. (2000). Development of a package in the Ox environment for GARCH models and two empirical applications. Mimeo, Universite de Liege.

[32] Rosenblatt, M. (1952). Remarks on a Multivariate Transformation, The Annals of Mathematical Statistics 23, 470.472 .

[33] Schwert, W. (1990). Stock Volatility and the Crash of '87'. Review of Financial Studies 3, 77102.

[34] Taylor, S. (1986). Modelling financial time series. Wiley, New York. 
472 CHOOSING THE BEST PERFORMING GARCH MODEL FOR SRI LANKA STOCK MARKET BY

NON-PARAMETRIC SPECIFICATION TEST

[35] Vlaar, P. and Palm, F. (1993). The message in weekly exchange rates in the European monetary system: mean reversion, conditional heteroskedasticity and jumps. Journal of Business and Economic Statistics 11, 351-360.

[36] Zakoian, J.M. (1994). Threshold Heteroscedastic Models. Journal of Economic Dynamics and Control 18, 931-955.

Received March 15, 2013; accepted November 10, 2013.

\author{
Aboobacker Jahufer \\ Department of Mathematical Sciences \\ Faculty of Applied Sciences \\ South Eastern University of Sri Lanka \\ Sri Lanka \\ jahufer@yahoo.com, jahufer@seu.ac.lk
}

